\title{
Ultrafast formation of the charge-transfer state of prodan reveals unique aspects of the chromophore environment
}

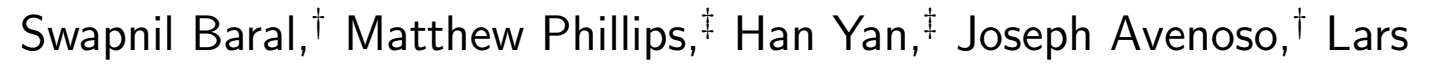 \\ Gundlach, ${ }^{\ddagger}, \dagger$ Björn Baumeier, $₫$ and Edward Lyman ${ }^{*,+, \dagger}$ \\ $\dagger$ Department of Physics and Astronomy, University of Delaware, Newark, DE 19716 USA \\ $\ddagger$ Department of Chemistry and Biochemistry, University of Delaware, Newark, DE 19716 \\ $U S A$ \\ IDepartment of Mathematics and Computer Science 83 Institute for Complex Molecular \\ System, P.O. Box 513, 5600 MB Eindhoven, The Netherlands \\ E-mail: elyman@udel.edu
}

\begin{abstract}
Lipiophilic dyes such as Laurdan and Prodan are widely used in membrane biology due to a strong bathochromic shift in emission that reports structural parameters of the membrane such as area per molecule. Disentangling the factors which control the spectral shift is complicated by the stabilization of a charge-transfer-like excitation of the dye in polar environments. Predicting the emission therefore requires modeling both the relaxation of the environment and the corresponding evolution of the excited state. Here an approach is presented in which (i) the local environment is sampled by classical molecular dynamics (MD) simulation of the dye and solvent; (ii) the electronically excited state of Prodan upon light absorption is predicted by numerical quantum
\end{abstract}


mechanics (QM); (iii) iterative relaxation of the environment around the excited dye by MD coupled with evolution of the excited state is performed; (iv) the emission properties are predicted by QM. The QM steps are computed using many-body Green's function in the $G W$ approximation and the Bethe-Salpeter Equation with the environment modeled as fixed point charges, sampled in the MD simulation steps. Comparison to ultrafast time resolved transient absorption measurements demonstrates that the iterative MM/QM approach agrees quantitatively with both the polarity dependent shift in emission and the timescale over which the charge transfer state is stabilized. Together the simulations and experimental measurements suggest that evolution into the charge-transfer state is slower in amphiphilic solvents.

\section{Introduction}

Polarity-sensitive dyes, 4-(2-(6-(dibutylamino)-2-naphthalenyl)ethenyl)-1 -(3-sulfopropyl) -hydroxide (di-4-ANEPPS), and the naphthalene derivatives 2-dimethylamino-6-propionylnaphthalene (Prodan), 2 -dimethylamino 6 -lauroylnaphthalene (Laurdan), and C-Laurdan

are widely used as reporters of membrane structure in both model and cell membranes. ${ }^{1,2}$ All are lipophilic, so that they partition to the membrane, and have spectral properties that depend on membrane structure, including lipid packing, membrane thickness, and chain ordering. The sensitivity of the Stokes shift of the Prodan family is especially dramatic, with a difference of $50 \mathrm{~nm}$ when comparing emission in a highly ordered and tightly-packed gel-phase membrane to a less ordered fluid membrane. ${ }^{1}$ This strong signal, together with tolerable toxicity underlie an enormous literature using these dyes to study membrane structure over the past 40 years. $^{1-3}$

The pronounced Stokes shift of the Prodan-derived dyes is due to a transition from an excitonic state to a charge transfer (CT) state that is extremely sensitive to the electronic environment. ${ }^{4}$ Applications to biomembranes have traditionally relied on a comparison of the relative fluorescence intensity at two different wavelengths (termed "generalized polar- 
ization", or GP) because the implementation is straightforward and is easily combined with confocal microscopy. ${ }^{1,5}$ More recently, methods have been introduced that make use of the full fluorescence spectrum ${ }^{6}$ and of the fluorescence lifetime (phasor methods). ${ }^{7}$ There have been some attempts to elucidate the excited state dynamics employing ultrafast spectroscopic methods. ${ }^{8}$ The stabilization of the charge transfer state may include changes in both hydrogen bonding ${ }^{9}$ and local polarization ${ }^{10}$ due to solvent reorientation, complicating an unambiguous interpretation of the experimental results.

Prior numerical work on the spectral properties of Prodan (Fig. 1) and related dyes have used time-dependent density functional theory (TD-DFT) with a continuum treatment of the environment. ${ }^{11-18}$ Although these methods give results in qualitative agreement with polarity-dependent spectroscopies, they fall short in two important respects. First, conventional DFT functionals are not well-suited to CT states due to the lack of proper long-range interactions, ${ }^{19}$ unless special system-tuned corrections are taken into account. ${ }^{20}$ Second, a continuum treatment of the environment will not capture the relaxation dynamics and coupled stabilization of the $\mathrm{CT}$ excited state.

In the following, ultrafast spectroscopy of Prodan is combined with state-of-the-art molecular dynamics/quantum mechanics simulations in order to resolve the time-evolution of the excited state, overcoming the limitations of previous work. Femtosecond transient absorption of Prodan in different solvents is applied to measure the solvent-dependent sub-picosecond formation of the CT state. The optical excitations of the dye are treated using Many-Body Green's Functions ${ }^{21}$ within the $G W$ approximation ${ }^{21}$ and the Bethe-Salpeter Equation ${ }^{22}$ ( $G W$-BSE), which yields accurate descriptions of localized excitonic and CT excitations on an equal footing, without the need for system- and environment-specific adjustments. ${ }^{23-25}$

An iterative molecular dynamics $/ G W$-BSE scheme is used to alternately relax the solvent environment and evolve the excited state. Excellent agreement between the simulated and measured spectral dynamics provides confidence in the integrated experimental/numerical approach, and promises reliable interpretation of spectra in more complex samples. The 
femtosecond absorption measurements reveal a new feature of the excited state dynamics: There are two relaxation processes (ps and tens of ps) in hydrocarbon alcohol solvents like octanol and methanol, but only a single fast relaxation in solvents like hexane, acetone, and water. This feature appears to be polarity-independent, and is quantitatively corroborated by the calculations. This observation requires the ultrafast methods employed in the present work, demonstrating the complementarity of the approach to earlier measurements using other types of time-dependent spectroscopies. ${ }^{6,26,27}$ The sensitivity of the relaxation dynamics to the local molecular environment suggests that the approach will yield new information on the local environment of polarity sensitive dyes when applied to biomembranes.

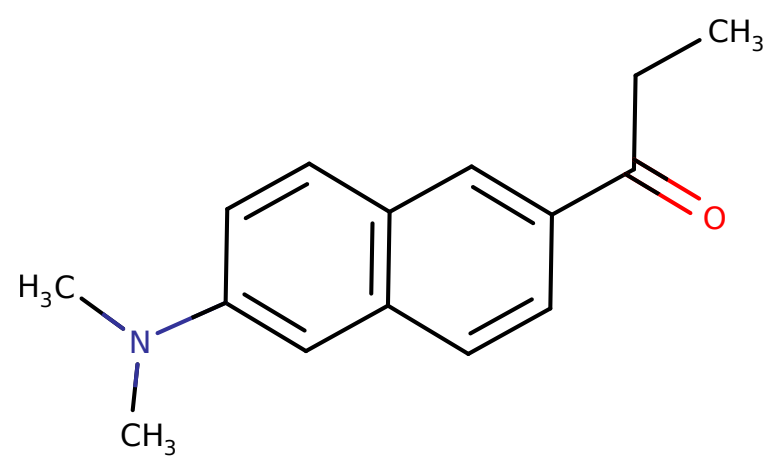

Figure 1: Chemical structure of 2-dimethylamino-6-propionylnaphthalene (Prodan)

\section{Methods}

\subsection{Theory}

Electronic excitations of a closed-shell system with net spin of zero are calculated using $G W$ BSE. This approach builds upon a DFT ${ }^{36}$ ground state calculation in which Kohn-Sham (KS) energy levels $\epsilon_{i}^{\mathrm{KS}}$ and wave functions $\left|\phi_{i}^{\mathrm{KS}}\right\rangle$ are obtained as solutions to

$$
\left[\hat{h}_{0}+\hat{V}_{\mathrm{XC}}\right]\left|\phi_{i}^{\mathrm{KS}}\right\rangle=\epsilon_{i}^{\mathrm{KS}}\left|\phi_{i}^{\mathrm{KS}}\right\rangle,
$$


Table 1: Prodan dipole moments reported in the literature. ${ }^{28-35} \mu_{\mathrm{G}}$ and $\mu_{\mathrm{E}}$ are ground state and excited state dipole moment of Prodan measured in Debye (D).

\begin{tabular}{lcc}
\hline Reference & $\mu_{G}(\mathrm{D})$ & $\mu_{E}(\mathrm{D})$ \\
\hline Balter et al. ${ }^{28}$ & 2.9 & 10.9 \\
Catalan et al. ${ }^{29}$ & 4.7 & 11.7 \\
Bunker et al. ${ }^{30}$ & 2.85 & 9.8 \\
Kawski ${ }^{31}$ & $2.1 / 2.5$ & $6.4 / 7.4$ \\
Kawski ${ }^{35}$ & $2.14 / 2.46$ & $6.46 / 7.37$ \\
Kawski et al. ${ }^{34}$ & $2.45 / 2.80$ & $6.65 / 7.6$ \\
Samanta, Fessenden ${ }^{32}$ & 5.2 & 10.2 \\
Cintia et al. ${ }^{33}$ & 5.5 & 20.0 \\
\hline
\end{tabular}

where $\hat{h}_{0}=\hat{T}_{0}+\hat{V}_{\text {ext }}+\hat{V}_{\mathrm{H}}$. $\hat{T}_{0}$ is the kinetic energy operator, $\hat{V}_{\text {ext }}$ the external potential, $\hat{V}_{\mathrm{H}}$ the Hartree potential, and $\hat{V}_{\text {XC }}$ the exchange-correlation potential. ${ }^{37}$

\subsubsection{Quasi-Particle Excitation}

First, quasi-particle (QP) states representing independent single-particle (electron or hole) excitations are calculated within the $G W$ approximation $^{21}$ from

$$
\left[\hat{h}_{0}+\hat{\Sigma}\left(\epsilon_{i}^{\mathrm{QP}}\right)\right]\left|\phi_{i}^{\mathrm{QP}}\right\rangle=\epsilon_{i}^{\mathrm{QP}}\left|\phi_{i}^{\mathrm{QP}}\right\rangle
$$

where $\left|\phi_{i}^{\mathrm{QP}}\right\rangle$ are QP wave functions and $\epsilon_{i}^{\mathrm{QP}}$ are the excitation energies of quasi-electron and quasi-hole states. In Eq. (2), the approximate exchange-correlation potential of KS-DFT as in Eq. (1) has been replaced by the self-energy $\hat{\Sigma}(E)$ which is a non-local, energy-dependent operator. Within the $G W$ approximation it is calculated as

$$
\hat{\Sigma}=i G W
$$

which is a convolution of the single-particle Green's function $G$ and the screened Coulomb interaction $W=\epsilon^{-1} v_{\mathrm{c}}$, where $v_{\mathrm{c}}=\left|\mathbf{r}-\mathbf{r}^{\prime}\right|^{-1}$ is the bare Coulomb interaction and $\epsilon\left(\mathbf{r}, \mathbf{r}^{\prime}, \omega\right)$ is the microscopic dielectric function calculated within the random-phase approximation. 
These quasi-electron and quasi-hole states are typically expanded in terms of KS states as

$$
\left|\phi_{i}^{\mathrm{QP}}\right\rangle=\sum_{j} C_{i j}\left|\phi_{j}^{\mathrm{KS}}\right\rangle
$$

Assuming that the Kohn-Sham states approximate the $G W$ quasi-particle states, i.e., $\left|\phi_{i}^{\mathrm{QP}}\right\rangle \approx\left|\phi_{i}^{\mathrm{KS}}\right\rangle$, the quasi-particle energies can be obtained perturbatively ${ }^{25}$ according to

$$
\begin{aligned}
\epsilon_{i}^{\mathrm{QP}} & =\epsilon_{i}^{\mathrm{KS}}+\Delta \epsilon_{i}^{\mathrm{QP}} \\
& =\epsilon_{i}^{\mathrm{KS}}+\left\langle\phi_{i}^{\mathrm{KS}}\left|\hat{\Sigma}\left(\epsilon_{i}^{\mathrm{QP}}\right)-\hat{V}_{\mathrm{XC}}\right| \phi_{i}^{\mathrm{KS}}\right\rangle .
\end{aligned}
$$

In Eq. (5), both the correction term $\Delta \epsilon_{i}^{\mathrm{QP}}$ and $\hat{\Sigma}$ (via $G$ and $W$ ) depend on $\epsilon_{i}^{\mathrm{QP}}$, requiring an iterative procedure until self-consistency in the eigenvalues $\epsilon_{i}^{\mathrm{QP}}$ is reached.

\subsubsection{Coupled Electron-Hole Excitations}

Quasi-particle excitations as above correspond to charged excitations of the system. Neutral excitations which involve a coupled electron-hole pair, e.g., after the absorption of a photon, are not included in this framework and need to be described using a two-particle wavefunction. ${ }^{25,38}$ The $S$-th excitation, $\chi_{S}$, can be written as the linear combination of quasi-particle product states as

$$
\chi_{S}\left(\mathbf{r}_{h}, \mathbf{r}_{e}\right)=A_{v c}^{S} \phi_{v}\left(\mathbf{r}_{h}\right) \phi_{c}^{*}\left(\mathbf{r}_{e}\right)+B_{v c}^{S} \phi_{c}\left(\mathbf{r}_{h}\right) \phi_{v}^{*}\left(\mathbf{r}_{e}\right),
$$

where $\mathbf{r}_{e}\left(\mathbf{r}_{h}\right)$ are the electron (hole) coordinates, $v$ and $c$ run over occupied and unoccupied single particle states respectively, and $A_{v c}^{S}\left(B_{v c}^{S}\right)$ are the (anti-)resonant electron-hole amplitudes. They and the associated excitation energy $\Omega_{S}$ are obtained by solving the BetheSalpeter equation, which can be cast in the form of a non-Hermitian eigenvalue problem

$$
\hat{H}^{\mathrm{BSE}}\left|\chi_{S}\right\rangle=\Omega_{S}\left|\chi_{S}\right\rangle
$$


or in matrix form

$$
\left(\begin{array}{cc}
H^{\mathrm{res}} & K \\
-K & -H^{\mathrm{res}}
\end{array}\right)\left(\begin{array}{c}
A^{S} \\
B^{S}
\end{array}\right)=\Omega_{S}\left(\begin{array}{c}
A^{S} \\
B^{S}
\end{array}\right)
$$

For spin-singlet excitations as considered in this work, the diagonal and off-diagonal blocks of the Hamiltonian in Eq. (8) are determined in the non-interacting quasi-particle basis according to

$$
\begin{aligned}
& H_{v c, v^{\prime} c^{\prime}}^{\mathrm{res}}=D_{v c, v^{\prime} c^{\prime}}+2 H_{v c, v^{\prime} c^{\prime}}^{\mathrm{x}}+H_{v c, v^{\prime} c^{\prime}}^{\mathrm{d}} \\
& K_{c v, v^{\prime} c^{\prime}}=2 H_{c v, v^{\prime} c^{\prime}}^{\mathrm{x}}+H_{c v, v^{\prime} c^{\prime}}^{\mathrm{d}}
\end{aligned}
$$

with

$$
\begin{aligned}
& D_{v c, v^{\prime} c^{\prime}}=\left(\epsilon_{v}-\epsilon_{c}\right) \delta_{v, v^{\prime}} \delta_{c, c^{\prime}} \\
& H_{v c, v^{\prime} c^{\prime}}^{\mathrm{x}}=\int d^{3} \mathbf{r}_{h} d^{3} \phi_{c}^{*}\left(\mathbf{r}_{e}\right) \phi_{v}\left(\mathbf{r}_{e}\right) \nu\left(\mathbf{r}_{e}, \mathbf{r}_{h}\right) \phi_{c^{\prime}}\left(\mathbf{r}_{h}\right) \phi_{v^{\prime}}^{*}\left(\mathbf{r}_{h}\right) \\
& H_{v c, v^{\prime} c^{\prime}}^{\mathrm{d}} \int d^{3} \mathbf{r}_{e} d^{3} \mathbf{r}_{h} \phi_{c}^{*}\left(\mathbf{r}_{e}\right) \phi_{c^{\prime}}\left(\mathbf{r}_{e}\right) W\left(\mathbf{r}_{e}, \mathbf{r}_{h}, \omega=0\right) \\
& \quad \times \phi_{v}\left(\mathbf{r}_{h}\right) \phi_{v^{\prime}}^{*}\left(\mathbf{r}_{h}\right) .
\end{aligned}
$$

The contribution $H^{x}$ is the repulsive exchange interaction originating from the unscreened interaction $v_{\mathrm{c}}$, while the direct interaction $H^{d}$ contains the attractive, but screened $(W)$, interaction between electron and hole and causes the binding of the electron hole pair. Furthermore, it is assumed here that the dynamic properties of $W(\omega)$ are negligible and the computationally less demanding static approximation $\omega=0$ is sufficient.

\subsubsection{Electrostatic embedding}

As discussed in the introduction, the optical excitations of Prodan are influenced by the structural details of the extended solvation shell. Emission involves coupled electronic and structural relaxation, which occurs on a timescale of ps and involves more nuclei than can be treated quantum mechanically. For this reason, a mixed approach is used, in which 
the excited state of the dye is calculated within $G W$-BSE embedded in the electrostatic background of the solvent sampled by classical molecular dynamics. The coupling of the solvent and the solute in the evaluation of the excitations within $G W$-BSE is performed using a molecular mechanics (MM) representation of the electrostatic potential. Specifically, the solvent molecules in the MM region are represented by the static atomic point charges $Q^{a}$ at positions $\mathbf{R}_{a}$ where $a$ indicates the atoms in the MM region. The classical electrostatic potential

$$
V_{\mathrm{MM}}(\mathbf{r})=\sum_{a \in \mathrm{MM}} \frac{Q^{a}}{\left|\mathbf{r}-\mathbf{R}_{a}\right|}
$$

from the MM region is added to $\hat{V}_{\text {ext }}$ in $\hat{h}_{0}$ in Eqs. (1) and (2), respectively.

Relaxation of the environment and corresponding evolution of the excited state is handled by an iterative process described in the next section.

\subsection{Computational Method}

\subsubsection{Classical MD}

Partial charges for the Prodan ground state have been obtained following the standard procedure for the CHARMM family of force fields. ${ }^{39,40}$ Initial guesses for partial charges were obtained from the CHARMM General Force Field (CGenFF) ${ }^{39}$ via the Paramchem web server. ${ }^{41,42}$ The initial charges did not yield a ground-state dipole moment in agreement with available data, and so partial charge optimization was performed. The geometry was optimized at the MP2/6-31G* level. ${ }^{43}$ Single-point energies for interactions with water were computed for TIP3P water molecules at several locations at the HF/6-31G* level. Water

interaction energies are scaled by factor of 1.16 to be relevant for the bulk phase. ${ }^{39}$ The level of theory and the scaling of energy is done to maintain the compatibility with CHARMM's additive force fields. Scaled $\mathrm{HF} / 6-31 \mathrm{G}^{*}$ model compound-water interaction energies and the computed dipole moment are used as target data, and the partial charges are adjusted until a best-fit between the QM and MM computed interaction energies and dipole moment is 
obtained. See supplemental information for more details, including the final values of the partial charges following optimization.

Six different systems were built with Packmol ${ }^{44}$ each of which included a single Prodan molecule in six different bulk solvents: 7200 TIP3P water, 2225 ethanol, 1770 acetone, 826 octanol, 3212 methanol, or 990 hexane. All MD simulations were run with GROMACS 5.1.1 ${ }^{45}$ using the CHARMM36 force field. ${ }^{39,40}$ Steepest descent energy minimization was followed by 20 ns NVT (Nose-Hoover ${ }^{46}$ for temperature coupling at 298K), and then $30 \mathrm{~ns}$ NPT equlibration (Berendsen ${ }^{47}$ for temperature and isotropic Parrinello-Rahman ${ }^{48}$ pressure coupling for $298 \mathrm{~K}$ and 1 atm pressure respectively), then $300 \mathrm{~ns}$ production simulation (Nose-Hoover temperature coupling ${ }^{46}$ and Parrinello-Rahman pressure coupling ${ }^{48}$ ). Lennard-Jones ${ }^{49}$ interactions were cut off using a switching function between $10 \AA$ and $12 \AA$ and particle mesh Ewald ${ }^{50}$ method with $12 \AA$ cutoff radius was used for long range electrostatics.

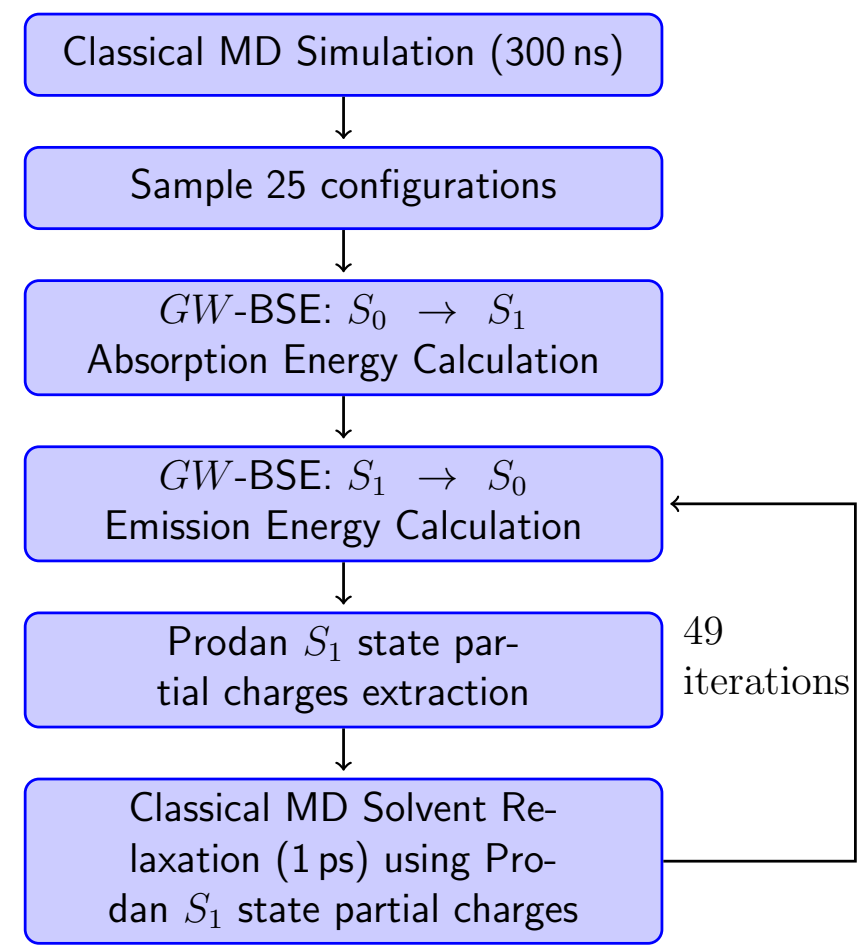

Figure 2: Prodan excitation and solvent relaxation workflow. 


\subsubsection{Excited state calculation and Iterative solvent relaxation}

$G W$-BSE calculations for singlet excited states were run using VOTCA-XTP ${ }^{25}$ which provides the interface to run Gaussian09 for the preceding ground state DFT calculation. All following results have been obtained based on DFT calculations using the PBE0 hybrid functional ${ }^{51}$ and the cc-pVTZ basis set and its optimized auxiliary basis ${ }^{52}$ to express twoelectron integrals within the resolution-of-identity technique. Seven hundred and forty eight KS orbitals are used within the RPA, and product functions for the BSE are formed using 61 (687) occupied (unoccupied) orbitals. The frequency dependence of the self-energy is evaluated using a generalized plasmon-pole model. ${ }^{53}$ Quasi-particle energies are determined self-consistently, as discussed above, until changes are smaller than $10^{-5}$ Hartree.

Special care needs to be taken to avoid spurious effects arising from inconsistencies of the classical and quantum molecular potential energy surface when $G W$-BSE calculations are performed on configurations sampled by classical MD. Deviations in equilibrium bond can change excitation energies by several tenths of an eV. VOTCA-XTP therefore post-processes the structures read from the MD trajectory and replaces the rigid parts of prodan, i.e., its naphthalene core, the carbonyl and methylene groups, with copies optimized on PBE0/ccpVTZ level for the DFT $S_{0}$ ground state (for absorption) and $G W$-BSE $S_{1}$ excited state (for emission). For information about ground state and excited state geometries, see Table S1 in supplementary material.

The MM/GW-BSE absorption and emission calculations are performed as shown in the flow chart in Fig. 2. First, twenty five configurations are randomly sampled from the $300 \mathrm{~ns}$ classical NPT MD simulation of Prodan in bulk solvents. Absorption energy from $S_{0} \rightarrow S_{1}$ state is computed as described in Sec. 2.2.2. Then, the initial calculation of the $S_{1} \rightarrow S_{0}$ emission energy is performed for the same twenty-five configurations. For each, the partial charges of the $S_{1}$ state are determined with the CHELPG method by constraining the partial charges of terminal hydrogens to $0.09 \mathrm{e}$ where e is the elementary charge and are then used to continue classical MD for another $1 \mathrm{ps}$ to relax the solvent environment in the presence 
of the changed dipole moment of the dye. The $G W$-BSE emission calculation is performed again in the new solvent background, and the procedure is repeated for fifty iterations.

\subsection{Experimental Methods}

Prodan was obtained from Invitrogen, and all solutions for transient absorption (TA) measurements were saturated. Spectroscopic grade solvents obtained from Fisher Chemicals and were dried over sodium sulfate before use.

1.25 micromolar solutions of Prodan were prepared from a stock solution in acetone: After allocation, the acetone was allowed to dry off in open air (about 30 seconds, aliquot was 50 microliters) leaving Prodan in the vial, to which the desired solvent was added and shaken well. This was done immediately before measurement for each solution. A scanning fluorometer (Fluoromax-4, Horiba) with excitation at 350 and $410 \mathrm{~nm}$ and a resolution of $5 \mathrm{~nm}$ was used. The spectra were recorded with a resolution of $1 \mathrm{~nm}$. To ensure the system was working properly and consistently a water blank was measured before and after experimental measurements, and the emission peak at $397 \mathrm{~nm}$ was observed with consistent wavelength and intensity.

Femtosecond transient spectra were recorded using a setup previously discussed. ${ }^{54}$ Briefly, the system is based on a $10 \mathrm{kHz}$ regenerative Ti:sapphire amplifier. White-light was generated in a $1 \mathrm{~mm}$ sapphire window and compressed to a group velocity mismatch of less than 2 fs across the spectrum using chirped-mirrors (Laser Quantum) and fused silica wedges. The pump pulse at $340 \mathrm{~nm}(3.65 \mathrm{eV})$ was generated by second harmonic generation from the output of a non-collinear parametric amplifier. The instrument response function of the setup was 25 fs.

Transient maps (Fig. 3 and SI Fig. S5 and S6) were analyzed by fitting spectra at a range of time steps to a Gaussian function. The peak maximum was plotted as a function of time. The shift of the maximum with time was fitted by one or two exponential functions to extract time-constants. 


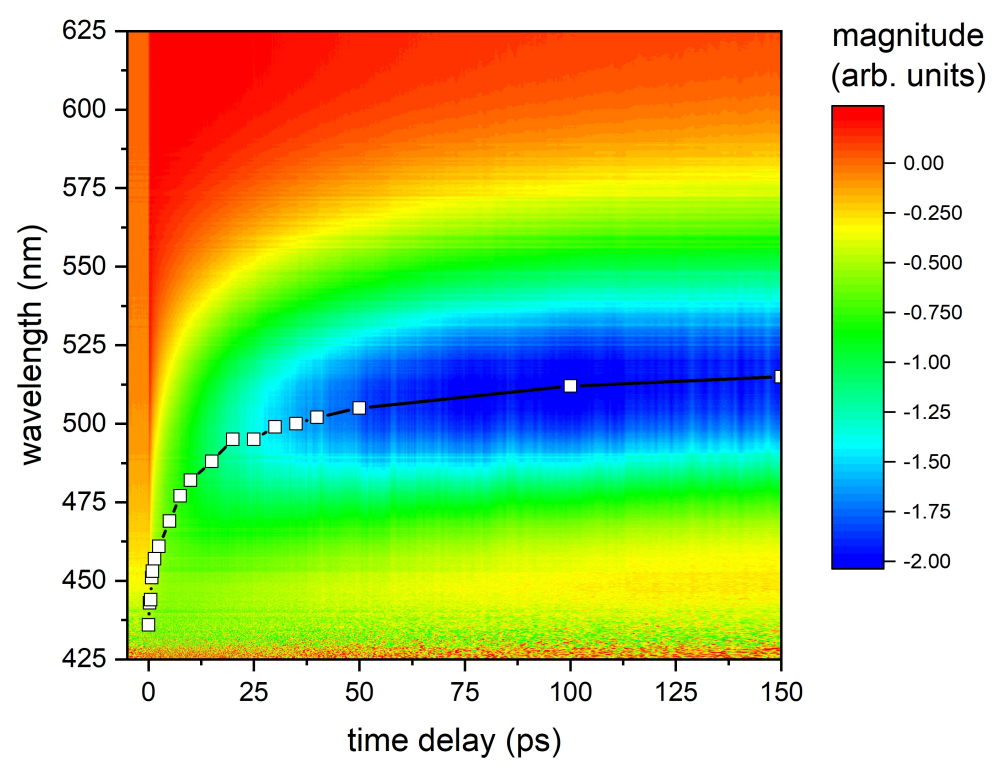

Figure 3: Prodan TA-map in ethanol. The black line indicates the maximum of the stimulated emission peak.

\section{Results}

\subsection{Simulation Results: Prodan emission and dipolar evolution in six different solvents}

Figure 4 shows the evolution of the $S_{1}$ state dipole moment of Prodan (a) and $S_{1}$ to $S_{0}$ emission energy (b) for the fifty steps of the iterative relaxation procedure in six different solvents of differing polarity.

In every case, both the excited state dipole and the emission energy plateau after (at most) $20 \mathrm{ps}$, indicating convergence of the iterative MM/GW-BSE relaxation protocol. During the 1 ps MD simulation steps, the solvent nuclear degrees of freedom relax in response to the excited state partial charges. In the least polar solvent (hexane), the dipole moment of the dye in the S1 state changes from 5.5 to 10.98 Debye. In more polar solvents the dipole moment evolves to a larger value (24 Debye in the most polar environment): The excited state dipole polarizes the environment, which stabilizes the charge transfer state, which polarizes the environment more. This process continues until the environment can no longer polarize. 

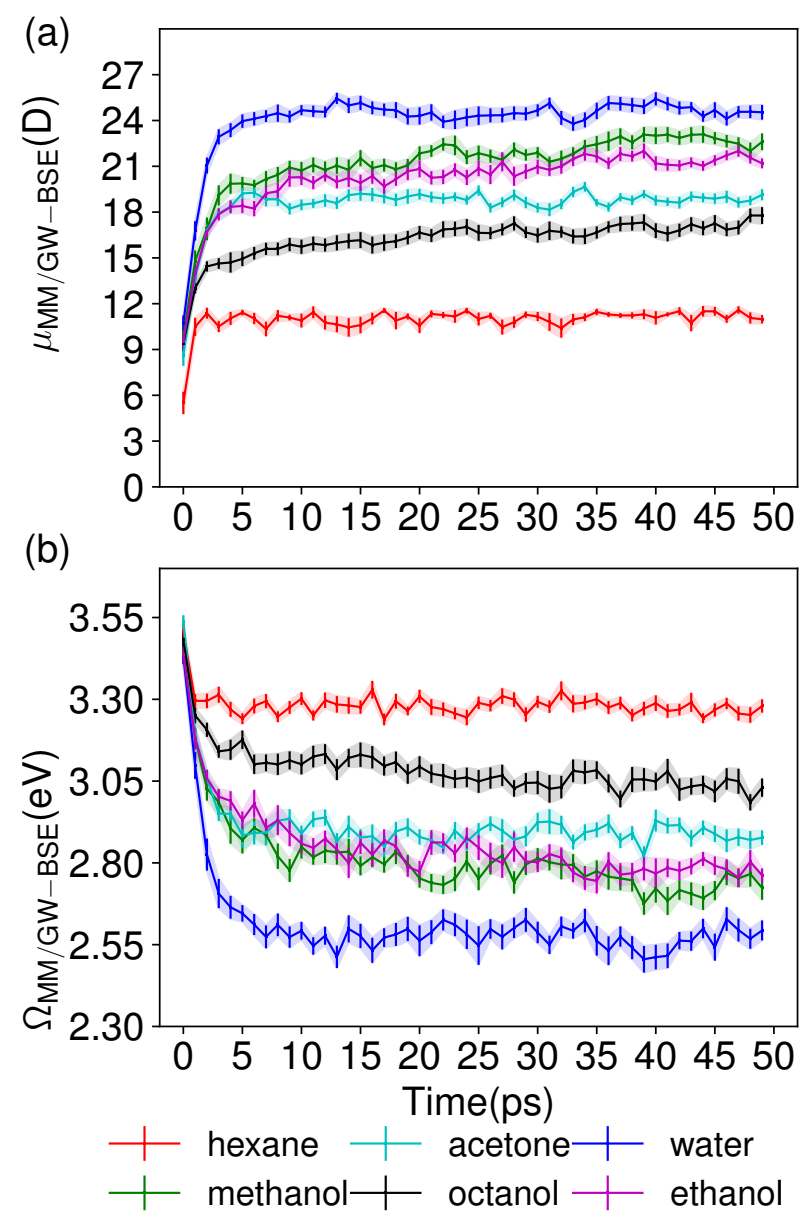

Figure 4: (a) Prodan $S_{1}$ state dipole moment and (b) $S_{1} \rightarrow S_{0}$ emission energy in hexane (red), octanol (black), acetone (cyan), ethanol (magenta), methanol (green), and water (blue). Error bars and background shading indicate the standard deviation among 25 different solvent configurations at each time point.

The predicted dipole moments for the CT state are within the range reported in the literature for the excited state (see Table 1), although the most polarizable environments yield dipole moments at the very upper end of this range. The dipole moments included in the table are obtained via different methods (solvatochromic shift, solvent pertubation, thermochromic shift, and microwave absorption) with the Onsager cavity radius approximated between 4.2 to $6.3 \AA$.

The observable effect of the stabilization of the CT state is the shift to lower emission energies with increasing polarity of the solvent, shown in Figure 4(b). After the solvent has 


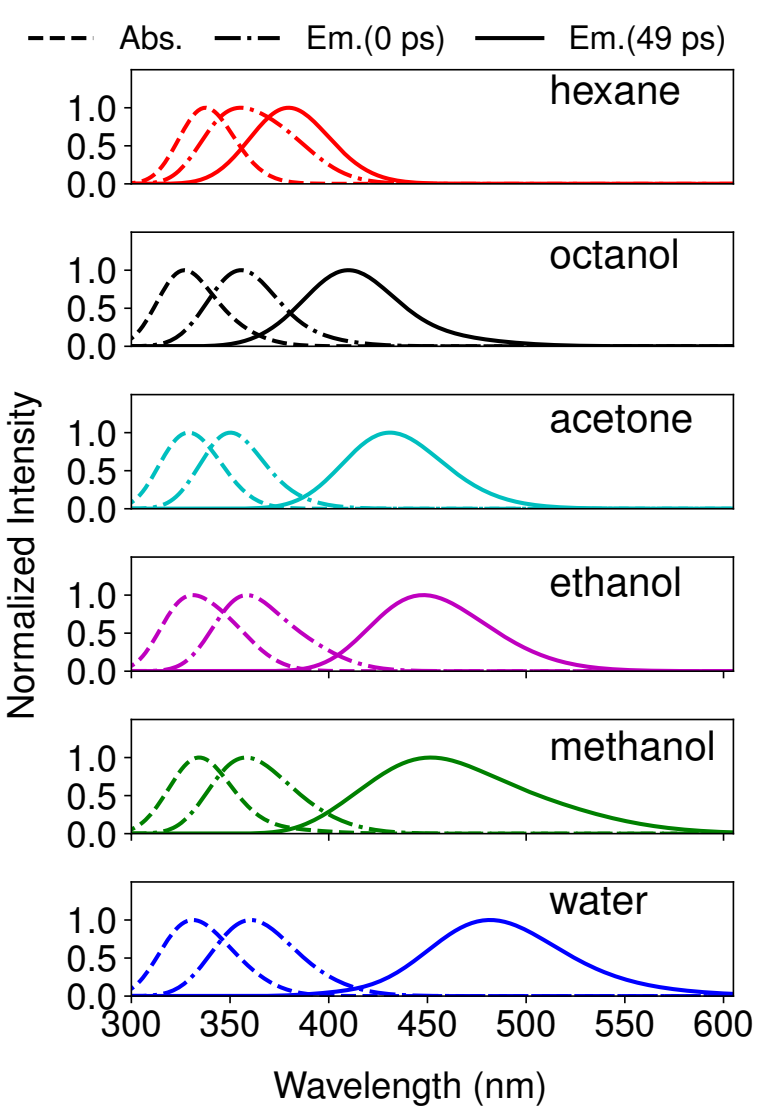

Figure 5: Normalized absorption (dashed lines), emission immediately after absorption (dashed-dotted lines) and after $49 \mathrm{ps} \mathrm{MM} / G W$-BSE relaxation (solid lines) spectra of Prodan in different solvents.

fully relaxed and the data have plateaued, the $S_{1}$ to $S_{0}$ emission energy predicts the steady state emission spectra that is typically reported in the literature as a probe of the solvent environment of the chromophore. A comparison to reported values for the Stokes shift will be revisited in the Discussion.

Steady state absorption and emission spectra are predicted by broadening the $S_{0}$ to $S_{1}$ and $S_{1}$ to $S_{0}$ transitions assuming Gaussian band shape with $\sigma=0.4 \mathrm{eV}$, and then averaging the obtained energies for twenty-five different configurations. Figure 5 shows the absorption spectra, the emission spectrum immediately after absorption, and the emission spectrum following the $49 \mathrm{ps}$ of iterative relaxation shown in Fig. 4. As expected, the absorption spectra show almost no dependence on the solvent. Similarly, the emission immediately 

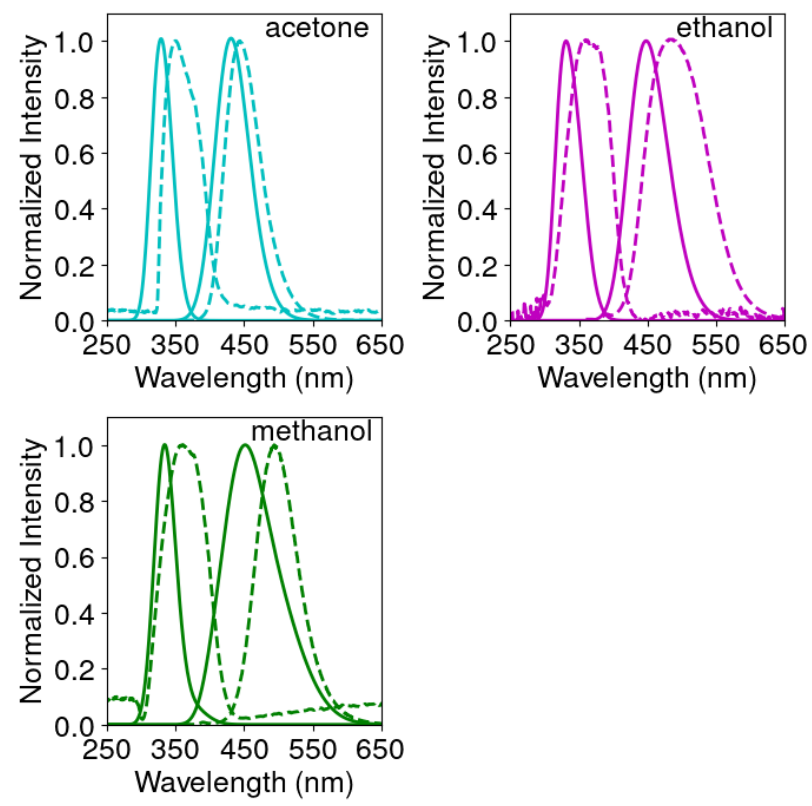

Figure 6: Prodan absorption (left) and emission (right) spectra in acetone, ethanol, and methanol from simulation (solid lines) and experimental measurements (dashed lines).

following excitation (and before the polar solvents can relax and stabilize the CT state) shows little dependence on the solvent environment. However, after the solvent is relaxed via the iterative series of MM/GW-BSE steps the emission in polar solvents displays a strong bathochromic shift as the CT state is progressively stabilized.

\subsection{Solvent relaxation dynamics: Comparison of ultrafast spec- troscopy to MM/GW-BSE approach}

Absorption and emission spectra of Prodan were measured in two solvents of similar polarity (acetone and methanol), but which differ in their ability to donate a hydrogen bond. (As mentioned in the Introduction, Prodan is a hydrogen bond acceptor (at the carbonyl), and this has an influence on spectral properties which is not captured in the present MM/GWBSE protocol. ${ }^{55,56}$ ) Figure 6 compares the absorption and emission spectra in acetone (aprotic) and methanol (h-bond donor) to the experimentally measured steady state spectra. No shift or fit parameters are applied to the predicted spectra. Although the absolute positions 

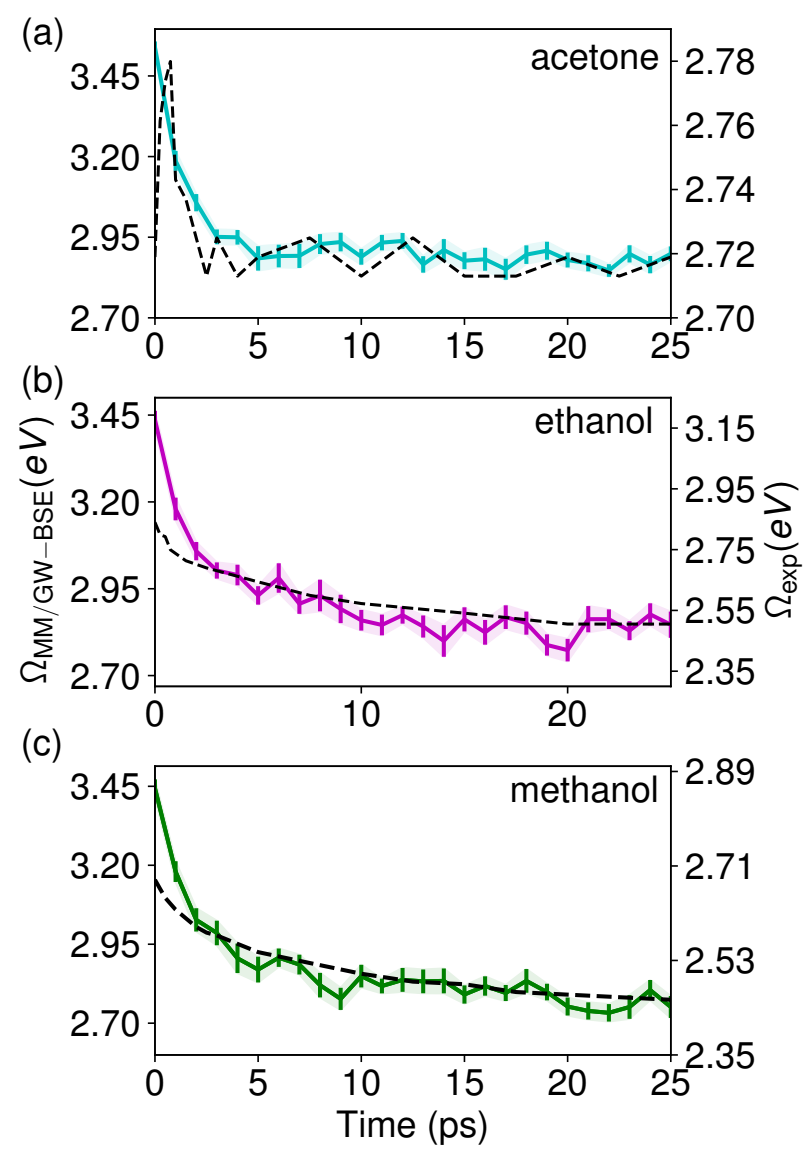

Figure 7: Prodan time resolved emission spectra (black) compared with simulation in acetone (cyan), ethanol (magenta), and methanol (green).

of the lines are at systematically higher energy in the MM/GW-BSE approach, the Stokes shifts are in nearly quantitative agreement, although the acetone calculation agrees slightly better $(94.0 \mathrm{~nm}$ (simulation) vs. $100.8 \mathrm{~nm}$ (experiment)) than the methanol calculation (117.6 nm calculated vs. $133.9 \mathrm{~nm}$ measured). Note that hydrogen bonding is not explicitly accounted for in the ab initio calculation.

Figure 7 compares the time-dependent relaxation of the environment obtained by the iterative MM/GW-BSE scheme to ultrafast measurements of the same. In both the experimental and simulated data, the relaxation dynamics in methanol are slower than in acetone. An exponential fit to the acetone data yields a single timescale of $2.63 \mathrm{ps}$ (experiment) and $1.36 \mathrm{ps}$ (simulation). The methanol data are better fit by a fast and a slow decay, with a timescale of $1.03 \mathrm{ps}$ and $15.16 \mathrm{ps}$ (experiment) and $1.74 \mathrm{ps}$ and $45.31 \mathrm{ps}$ (simulation). Fast 


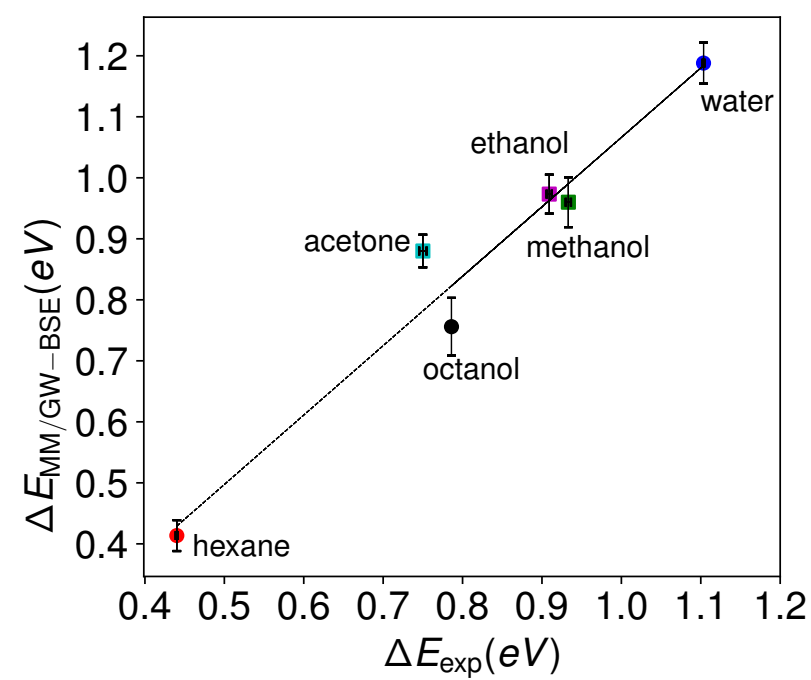

Figure 8: Comparison of Stokes shift obtained in simulation with experimental measurements from the literature ${ }^{55}(\bullet)$ and Figure $6(\mathbf{\bullet})$.

and slow timescales are also observed in ethanol, with timescales of $0.18 \mathrm{ps}$ and $13.44 \mathrm{ps}$ (experiment) and $1.25 \mathrm{ps}$ and $13.61 \mathrm{ps}$ (simulation).

The simulations confirm the observation of fast and slow relaxations in the alcohols, but not in acetone. Fitting the relaxation of the excited state for the rest of the simulation data reveals an unexpected trend: Three very different solvents (hexane, acetone, and water) all display a single, fast timescale. In contrast all of the hydrocarbon alcohols (octanol, ethanol, methanol) show a fast (ps) and slow (tens of ps) relaxation. This suggests that the slow relaxation is not due not to polarization of the environment, nor to formation of hydrogen bonds. Instead, the only commonality among the solvents with a second, slower timescale is that they are all weakly amphiphilic. We speculate that the slower timescale is due to local rearrangement of the solvent to form a slightly more hydrophilic cavity for the excited chromophore. It will be interesting to see how these observations carry over to ultrafast measurements in lipid environments, which embed the chromophore in a more chemically and dielectrically diverse interface. 
Table 2: Stokes shift of Prodan spectra from MM/GW-BSE calculated absorption and emission energies compared to experimental measurements from literature ${ }^{55}$ and Figure 6.

\begin{tabular}{lcccc}
\hline Solvent & $E_{\mathrm{MM} / G W \text {-BSE }}^{\text {absortion }}(\mathrm{eV})$ & $E_{\mathrm{MM} / G W \text {-BSE }}^{\text {emission }}(\mathrm{eV})$ & $\Delta E_{\mathrm{MM} / G W \text {-BSE }}(\mathrm{eV})$ & $\Delta E_{\text {exp }}(\mathrm{eV})$ \\
\hline Hexane & 3.67 & 3.26 & 0.41 & 0.44 \\
Acetone & 3.76 & 2.88 & 0.88 & 0.74 \\
Octanol & 3.79 & 3.03 & 0.76 & 0.79 \\
Ethanol & 3.75 & 2.77 & 0.98 & 0.91 \\
Methanol & 3.71 & 2.75 & 0.96 & 0.93 \\
Water & 3.75 & 2.57 & 1.18 & 1.10 \\
& & & &
\end{tabular}

Table 3: Prodan excited state relaxation times obtained from single and double exponential fits, respectively. For details about the fitting functions, see Supplemental Table S2. $\tau_{\mathrm{MM} / G W \text {-BSE }}$ and $\tau_{\text {exp }}$ are relaxation time from simulation and experiment, respectively.

\begin{tabular}{lcc}
\hline Solvent & $\tau_{\mathrm{MM} / G W \text {-BSE }}(\mathrm{ps})$ & $\tau_{\exp }(\mathrm{ps})$ \\
\hline Hexane & $0.41 \pm 0.19$ & $\mathrm{~N} / \mathrm{A}$ \\
Acetone & $1.36 \pm 0.11$ & $2.63 \pm 1.13$ \\
Water & $1.74 \pm 0.11$ & $\mathrm{~N} / \mathrm{A}$ \\
Octanol & $0.89 \pm 0.1$ & $\mathrm{~N} / \mathrm{A}$ \\
& $29.13 \pm 15.44$ & \\
Ethanol & $1.25 \pm 0.28$ & $0.18 \pm 0.04$ \\
& $13.61 \pm 3.96$ & $13.44 \pm 1.66$ \\
Methanol & $1.74 \pm 0.27$ & $1.03 \pm 0.11$ \\
& $45.31 \pm 54.33$ & $15.16 \pm 0.6$ \\
\hline
\end{tabular}

\section{Discussion}

Prediction of the spectral properties of polarity sensitive dyes by a combined MM/ $G W$-BSE approach yields excellent agreement with both steady state and time-resolved, ultrafast measurements. The iterative approach based on alternating MD relaxation of the solvent environment and GW-BSE prediction of the excited state leads to progressive stabilization of a charge-transfer excited state in more polar environments, resulting in lower energy emission as polarity increases. Comparison of the Stokes shift obtained for the six solvents in Figures 4 and 5 to results presented here and in the published literature ${ }^{55}$ yields an excellent, linear correlation with a slope of 1 (Figure 8), without any adjustment of the simulated data.

Ultrafast spectroscopy directly reveals the evolution of the CT state in polar environments 
(both protic and aprotic), providing evidence for a fast (ca. 1 ps) and slow (ca. 10-20 ps) process. Note that both of these processes are too fast to be observed by previous applications of other time-resolved spectroscopies to Prodan and related dyes. ${ }^{57-60}$ Remarkably, the iterative MM/GW-BSE approach also agrees quantitatively with timescale of relaxation in acetone, ethanol, and methanol, when the duration of the MD steps is appropriately chosen. (A longer solvent relaxation step arbitrarily increases the simulated relaxation timescale, and a shorter step does not change the observed timescales (see Supplemental Figure S4 and S5).

The iterative MM/GW-BSE approach presented here is designed specifically for quantitative prediction of the spectral properties of polarity sensitive dyes that are commonly used to study biomembrane structure and dynamics. The MD portion of the calculation (if the model is well parametrized) accounts for the heterogeneity of the environment, overcoming the limitations of continuum treatments that are more commonly used. The GW-BSE approach for excited state prediction is inherently better suited to CT states than TD-DFT approaches that have been previously applied to the same compounds. ${ }^{11-18}$ Together, these two components yield a very high accuracy, quantitative method with the potential to reveal the mechanism of other more recently developed, less well understood chromophores. Integrating the calculations with ultrafast measurements of the excited state dynamics will provide valuable information on the nature of the emitting state for these other dyes.

\section{Acknowledgments}

BB acknowledges support by the Innovational Research Incentives Scheme Vidi of the Netherlands Organisation for Scientific Research (NWO) with project number 723.016.002.

EL and SB were supported by the National Institutes of Health (R01GM116961). This

work used the Extreme Science and Engineering Discovery Environment (XSEDE) computing resource Stampede at the Texas Advanced Computing Center (TG-MCB170146), which 
is supported by National Science Foundation grant number ACI-1548562.

\section{References}

(1) Sanchez, S. A.; Tricerri, M. A.; Gunther, G.; Gratton, E.; Plata, N. D. L.; Plata, L. Modern Research and Educational Topics in Microscopy; 2007.

(2) Sanchez, S. A.; Tricerri, M. A.; Gratton, E. Laurdan Generalized Polarization Fluctuations Measures Membrane Packing Micro-Heterogeneity in Vivo. PNAS 2012, 109, $7314-7319$.

(3) Masukawa, M. K.; Vequi-Suplicy, C. C.; Duarte, E. L.; Lamy, M. T. A Closer Look into Laurdan as a Probe to Monitor Cationic DODAB Bilayers. Journal of Photochemistry and Photobiology A: Chemistry 2019, 376, 238-246.

(4) Lobo, B. C.; Abelt, C. J. Does PRODAN Possess a Planar or Twisted Charge-Transfer Excited State? Photophysical Properties of Two PRODAN Derivatives. J. Phys. Chem. A 2003, 10\%, 10938-10943.

(5) Parasassi, T.; De Stasio, G.; d'Ubaldo, A.; Gratton, E. Phase Fluctuation in Phospholipid Membranes Revealed by Laurdan Fluorescence. Biophysical Journal 1990, 57, $1179-1186$.

(6) Leonard, C.; Errachid, A.; Daubie, J.; Beghuin, D.; Courtois, P.-J.; MingeotLeclercq, M.-P.; Tyteca, D. Hyperspectral Analysis of Laurdan Emission Spectra in Red Blood Cells and Giant Unilamellar Vesicles. Biophysical Journal 2015, 108, 622a.

(7) Malacrida, L.; Jameson, D. M.; Gratton, E. A Multidimensional Phasor Approach Reveals LAURDAN Photophysics in NIH-3T3 Cell Membranes. Sci Rep 2017, 7.

(8) Weber, G.; Farris, F. J. Synthesis and Spectral Properties of a Hydrophobic Fluo- 
rescent Probe: 6-Propionyl-2-(Dimethylamino)Naphthalene. Biochemistry 1979, 18 , $3075-3078$.

(9) Vequi-Suplicy, C. C.; Coutinho, K.; Lamy, M. T. New Insights on the Fluorescent Emission Spectra of Prodan and Laurdan. J Fluoresc 2015, 25, 621-629.

(10) Parasassi, T.; Krasnowska, E. K.; Bagatolli, L.; Gratton, E. Laurdan and Prodan as Polarity-Sensitive Fluorescent Membrane Probes. Journal of Fluorescence 1998, $8,365-373$.

(11) Mennucci, B.; Caricato, M.; Ingrosso, F.; Cappelli, C.; Cammi, R.; Tomasi, J.; Scalmani, G.; Frisch, M. J. How the Environment Controls Absorption and Fluorescence Spectra of PRODAN: A Quantum-Mechanical Study in Homogeneous and Heterogeneous Media. J. Phys. Chem. B 2008, 112, 414-423.

(12) Zharkova, O. M.; Rakhimov, S. I.; Morozova, Y. P. Quantum-Chemical Investigation of the Spectral Properties of Fluorescent Probes Based on Naphthalene Derivatives (Prodan, Promen). Russ Phys J 2013, 56, 411-419.

(13) Fukuda, R.; Chidthong, R.; Cammi, R.; Ehara, M. Optical Absorption and Fluorescence of PRODAN in Solution: Quantum Chemical Study Based on the Symmetry-Adapted Cluster-Configuration Interaction Method. Chem. Phys. Lett. 2012, 552, 53-57.

(14) Zhao, D.; Liu, Y.; Zhang, X.; Gao, J.; Liu, S.; Zhao, Z. Theoretical Insights into the Excited-State Intermolecular Hydrogen Bonding Dynamics of PRODAN Derivative in Toluene Solution. Chem. Phys. Lett. 2019, 725, 109-113.

(15) Guido, C. A.; Knecht, S.; Kongsted, J.; Mennucci, B. Benchmarking Time-Dependent Density Functional Theory for Excited State Geometries of Organic Molecules in GasPhase and in Solution. J. Chem. Theory Comput. 2013, 9, 2209-2220. 
(16) Pederzoli, M.; Sobek, L.; Brabec, J.; Kowalski, K.; Cwiklik, L.; Pittner, J. Fluorescence of PRODAN in Water: A Computational QM/MM MD Study. Chem. Phys. Lett. 2014, $597,57-62$.

(17) Cwiklik, L.; Aquino, A. J. A.; Vazdar, M.; Jurkiewicz, P.; Pittner, J.; Hof, M.; Lischka, H. Absorption and Fluorescence of PRODAN in Phospholipid Bilayers: A Combined Quantum Mechanics and Classical Molecular Dynamics Study. J. Phys. Chem. A 2011, 115, 11428-11437.

(18) Uudsemaa, M.; Trummal, A.; de Reguardati, S.; Callis, P. R.; Rebane, A. TD-DFT Calculations of One- and Two-Photon Absorption in Coumarin C153 and Prodan: Attuning Theory to Experiment. Phys. Chem. Chem. Phys. 2017, 19, 28824-28833.

(19) Dreuw, A.; Head-Gordon, M. Failure of Time-Dependent Density Functional Theory for Long-Range Charge-Transfer Excited States: The Zincbacteriochlorin-Bacteriochlorin and Bacteriochlorophyll-Spheroidene Complexes. J. Am. Chem. Soc. 2004, 126, 40074016.

(20) Kümmel, S. Charge-Transfer Excitations: A Challenge for Time-Dependent Density Functional Theory That Has Been Met. Advanced Energy Materials 2017, 7, 1700440.

(21) Hedin, L. New Method for Calculating the One-Particle Green's Function with Application to the Electron-Gas Problem. Phys. Rev. 1965, 139, A796-A823.

(22) Salpeter, E. E.; Bethe, H. A. A Relativistic Equation for Bound-State Problems. Phys. Rev. 1951, 84, 1232-1242.

(23) Blase, X.; Attaccalite, C. Charge-Transfer Excitations in Molecular Donor-Acceptor Complexes within the Many-Body Bethe-Salpeter Approach. Appl. Phys. Lett. 2011, 99, 171909. 
(24) Baumeier, B.; Andrienko, D.; Rohlfing, M. Frenkel and Charge-Transfer Excitations in Donor-Acceptor Complexes from Many-Body Green's Functions Theory. J. Chem. Theory. Comput. 2012, 8, 2790-2795.

(25) Wehner, J.; Brombacher, L.; Brown, J.; Junghans, C.; Çaylak, O.; Khalak, Y.; Madhikar, P.; Tirimbò, G.; Baumeier, B. Electronic Excitations in Complex Molecular Environments: Many-Body Green's Functions Theory in VOTCA-XTP. J. Chem. Theory. Comput. 2018, 14, 6253-6268.

(26) Tiziana, Parasassi,; Filippo, Conti,; Enrico, Gratton, TIME-RESOLVED FLUORESCENCE EMISSION SPECTRA OF LAURDAN IN PHOSPHOLIPID VESICLES BY MULTIFREQUENCY PHASE AND MODULATION FLUOROMETRY. Cellular and Molecular Biology 1986,

(27) Rowe, B. A.; Neal, S. L. Photokinetic Analysis of PRODAN and LAURDAN in Large Unilamellar Vesicles from Multivariate Frequency-Domain Fluorescence. J. Phys. Chem. B 2006, 110, 15021-15028.

(28) Balter, A.; Nowak, W.; Pawełkiewicz, W.; Kowalczyk, A. Some Remarks on the Interpretation of the Spectral Properties of Prodan. Chem. Phys. Lett. 1988, 143, 565-570.

(29) Catalan, J.; Perez, P.; Laynez, J.; Blanco, F. G. Analysis of the Solvent Effect on the Photophysics Properties of 6-Propionyl-2-(Dimethylamino)Naphthalene (PRODAN). $J$ Fluoresc 1991, 1, 215-223.

(30) Bunker, C. E.; Bowen, T. L.; Sun, Y.-P. A Photophysical Study of Solvatochromic Probe 6-Propionyl-Z(n,n-Dimethylamino)Naphthalene (Prodan) in Solution. Photochemistry and Photobiology 1993, 58, 499-505.

(31) Kawski, A. Ground-and Excited-State Dipole Moments of 6-Propionyl-2(Dimethylamino)Naphthalene Determined from Solvatochromic Shifts. Zeitschrift Naturforschung Teil A 1999, 54, 379-381. 
(32) Samanta, A.; Fessenden, R. W. Excited State Dipole Moment of PRODAN as Determined from Transient Dielectric Loss Measurements. J. Phys. Chem. A 2000, 104, $8972-8975$.

(33) Vequi-Suplicy, C. C.; Coutinho, K.; Lamy, M. T. Electric Dipole Moments of the Fluorescent Probes Prodan and Laurdan: Experimental and Theoretical Evaluations. Biophys Rev 2014, 6, 63-74.

(34) Kawski, A.; Kukliński, B.; Bojarski, P. Thermochromic Shifts of Absorption and Fluorescence Spectra and Excited State Dipole Moment of PRODAN. Zeitschrift für Naturforschung A 2000, 55, 550-554.

(35) Kawski, A.; Kukliński, B.; Bojarski, P. Thermochromic Shifts of Absorption and Fluorescence Spectra and Excited State Dipole Moment of PRODAN. Zeitschrift für Naturforschung A 2000, 55, 550 - 554 .

(36) Kohn, W.; Sham, L. J. Self-Consistent Equations Including Exchange and Correlation Effects. Phys. Rev. 1965, 140, A1133-A1138.

(37) Onida, G.; Reining, L.; Rubio, A. Electronic Excitations: Density-Functional versus Many-Body Green's-Function Approaches. Rev. Mod. Phys. 2002, 74, 601.

(38) Rohlfing, M. Excited States of Molecules from Green's Function Perturbation Techniques. 2000.

(39) Vanommeslaeghe, K.; Hatcher, E.; Acharya, C.; Kundu, S.; Zhong, S.; Shim, J.; Darian, E.; Guvench, O.; Lopes, P.; Vorobyov, I.; Mackerell, A. D. CHARMM General Force Field: A Force Field for Drug-like Molecules Compatible with the CHARMM All-Atom Additive Biological Force Fields. J. Comput. Chem. 2010, 31, 671-690.

(40) Klauda, J. B.; Venable, R. M.; Freites, J. A.; O'Connor, J. W.; Tobias, D. J.; Mondragon-Ramirez, C.; Vorobyov, I.; MacKerell, A. D.; Pastor, R. W. Update of the 
CHARMM All-Atom Additive Force Field for Lipids: Validation on Six Lipid Types. J. Phys. Chem. B 2010, 114, 7830-7843.

(41) Vanommeslaeghe, K.; Raman, E. P.; MacKerell, A. D. Automation of the CHARMM General Force Field (CGenFF) II: Assignment of Bonded Parameters and Partial Atomic Charges. J. Chem. Inf. Model. 2012, 52, 3155-3168.

(42) Vanommeslaeghe, K.; MacKerell, A. D. Automation of the CHARMM General Force Field (CGenFF) I: Bond Perception and Atom Typing. J Chem Inf Model 2012, 52, $3144-3154$.

(43) Frisch, M. J. et al. Gaussian 09 Revision D.01. 2009,

(44) Martínez, L.; Andrade, R.; Birgin, E. G.; Martínez, J. M. PACKMOL: A package for building initial configurations for molecular dynamics simulations. J. Comput. Chem. 2009, 30, 2157-2164.

(45) Van Der Spoel, D.; Lindahl, E.; Hess, B.; Groenhof, G.; Mark, A. E.; Berendsen, H. J. C. GROMACS: Fast, Flexible, and Free. J. Comput. Chem. 2005, 26, 1701-1718.

(46) Hoover, W. Canonical Dynamics: Equilibrium Phase-Space Distributions. Phys. Rev. A: At., Mol., Opt. Phys. 1985, 31, 1695.

(47) Berendsen, H. J. C.; Postma, J. P. M.; van Gunsteren, W. F.; DiNola, A.; Haak, J. R. Molecular Dynamics with Coupling to an External Bath. J. Chem. Phys. 1984, 81, $3684-3690$.

(48) Parrinello, M. Polymorphic Transitions in Single Crystals: A New Molecular Dynamics Method. J. Appl. Phys. 1981, 52, 7182.

(49) Steinbach, P. J.; Brooks, B. R. New Spherical-Cutoff Methods for Long-Range Forces in Macromolecular Simulation. J. Comput. Chem. 1994, 15, 667-683. 
(50) Darden, T.; York, D.; Pedersen, L. Particle Mesh Ewald: An Nṡlog(N) Method for Ewald Sums in Large Systems. Journal of Chemical Physics 1993, 98, 10089-10092.

(51) Ernzerhof, M.; Scuseria, G. E. Assessment of the Perdew-Burke-Ernzerhof ExchangeCorrelation Functional. J. Chem. Phys. 1999, 110, 5029-5036.

(52) Weigend, F.; Köhn, A.; Hättig, C. Efficient Use of the Correlation Consistent Basis Sets in Resolution of the Identity MP2 Calculations. J. Chem. Phys. 2002, 116, 3175-3183.

(53) Rohlfing, M.; Krüger, P.; Pollmann, J. Efficient Scheme for GW Quasiparticle Band-Structure Calculations with Applications to Bulk $\mathrm{Si}$ and to the $\mathrm{Si}(001)$ (2\ifmmode $\backslash$ times $\backslash$ else $\backslash$ texttimes $\backslash$ fi \{\} 1$)$ Surface. Phys. Rev. B 1995, 52, 1905-1917.

(54) Abraham, B.; Nieto-Pescador, J.; Gundlach, L. Ultrafast Relaxation Dynamics of Photoexcited Zinc-Porphyrin: Electronic-Vibrational Coupling. J. Phys. Chem. Lett. 2016, 7, 3151-3156.

(55) Moyano, F.; Biasutti, M. A.; Silber, J. J.; Correa, N. M. New Insights on the Behavior of PRODAN in Homogeneous Media and in Large Unilamellar Vesicles. J. Phys. Chem. $B$ 2006, 110, 11838-11846.

(56) Rowe, B. A.; Roach, C. A.; Lin, J.; Asiago, V.; Dmitrenko, O.; Neal, S. L. Spectral Heterogeneity of PRODAN Fluorescence in Isotropic Solvents Revealed by Multivariate Photokinetic Analysis. J. Phys. Chem. A 2008, 112, 13402-13412.

(57) Karmakar, R.; Samanta, A. Steady-State and Time-Resolved Fluorescence Behavior of C153 and PRODAN in Room-Temperature Ionic Liquids. J. Phys. Chem. A 2002, 106, 6670-6675.

(58) Sýkora, J.; Kapusta, P.; Fidler, V.; Hof, M. On What Time Scale Does Solvent Relaxation in Phospholipid Bilayers Happen? Langmuir 2002, 18, 571-574. 
(59) Bright, F. V.; Munson, C. A. Time-Resolved Fluorescence Spectroscopy for Illuminating Complex Systems. Analytica Chimica Acta 2003, 500, 71-104.

(60) Hutterer, R.; Parusel, A. B. J.; Hof, M. Solvent Relaxation of Prodan and Patman: A Useful Tool for the Determination of Polarity and Rigidity Changes in Membranes. Journal of Fluorescence 1998, 8, 389-393. 
Graphical TOC Entry 